\title{
An Application of Gaussian Mixtures: Colour Segmenting for the Four Legged League using HSI Colour Space
}

\author{
Naomi Henderson, Robert King, and Richard H. Middleton \\ School of Electrical Engineering \& Computer Science \\ The University of Newcastle, Callaghan 2308, Australia \\ \{naomi.henderson, robert.king, richard.middleton\}@newcastle.edu. au
}

\begin{abstract}
In the colour coded environment of the RoboCup 4 Legged League it is crucial to extract as much colour information as possible from an image without error. To do this requires hours of manual YUV pixel mapping and testing to ensure robustness under all possible lighting conditions. The YUV colour space is a very convenient standard for transmission of video data, but for colour classification and segmentation it suffers from being non-intuitive and sensitive to changes in lighting. Alternatively, colour classification principles can be applied in an HSI colour space; one of the convenient characteristics of the HSI colour space is that the hue value, $\mathrm{H}$, represents the colour wavelength information. From this concept it is easier to separate and label colour regions in an automated process as the theoretical hue and colour wavelength relationship is known. By fitting a Gaussian model using mixtures to HSI histograms we can generate boundaries of colour classes in HSI colour space.
\end{abstract}

\section{Introduction}

Colour classification is the first stage of image processing in many RoboCup software systems. It is also the primary, or in many cases effectively the only, sensor for the robot. For this reason the calibration of colour information requires very careful attention. Currently manual colour calibration is still the most common process used during competition for its reliability. Manual colour calibration process involves hours of mapping image pixels to a colour key defined in a look up table (LUT), a three dimensional array referenced by $\mathrm{Y}, \mathrm{U}$ and $\mathrm{V}$ values. Automation of the colour calibration process would have significant impact on the human involvement required during RoboCup set up. Many methods have been used to automate the colour calibration process [1] [2] but often require a routine to gain the additional information needed to make a colour decision. However, by transforming the image from the YUV colour space to the HSI colour space we can use hue information to separate and label regions of object colours in colour space using Gaussian mixtures. This paper proposes a compromise between the common process of manual calibration and the uncertain performance 
of full automation by defining colour classes as a set of tunable upper and lower boundaries in the HSI colour space. The approach still allows for testing and manual tuning whilst greatly reducing calibration time and increasing reliability of the colour information.

Section 2 of this paper presents an overview of the vision system that the classification system is applied to. Section 3 presents the stages of the HSI calibration system which involves the collection of HSI histograms and the application of Gaussian mixtures to separate hue information for the generation of upper and lower boundaries in HSI colour space. Example images of the calibration system and results are outlined in Section 4 and future work involving multi dimensional Gaussian mixtures is outlined in Section 5.

\section{Background}

\subsection{Vision Processing}

Objects of the RoboCup Soccer environment are colour coded so that they can be uniquely identified by the region of colour space they occupy. In ideal situations, i.e. bright lighting and good quality camera, the YUV values for each object would remain constant and could therefore be directly mapped to a single hard colour [3]. However in the 4LL where there are hardware limitations and varied lighting of objects due to the dynamic environment, these regions of colour space tend to overlap making regions of colour space non unique and the previously defined YUV mapping becomes invalid [4]. A soft colour classification system was implemented as a practical solution to classifying this overlapping colour space, it classifies these regions as a 'soft' colour and delays the colour decision making process until the entire image is processed.

The input image of the robot vision system used follows the process; colour classification referencing a YUV LUT, blob formation, soft colour blob filtering and size modification, and object recognition[5]. This section details the colour classification system as it is an important aspect of the base vision system. The rest of the paper is focused on the off line colour calibration process involved with building the reference LUT.

\subsection{Soft Colour Classification}

In the 4LL we are concerned with identifying certain landmarks and objects that are uniquely colour coded. These objects and landmarks and their colour codes are; orange ball, yellow goal and beacons, sky blue goal and beacons, red robot uniforms, navy blue robot uniforms, green field, white robots and field lines. Additionally black occurs in images with robots, dark shadows and dark backgrounds. Certain colours are close in any colour space (YUV, HSI, RGB, etc) and hence more difficult to separate. For the purpose of the 4LL the pairs of close colours are; red and orange, orange and yellow, and dark green and dark blue. They are of concern as a shift in colour space due to a change in lighting 
causes an overlap of these classified regions for this reason the soft colours: redorange, yellow-orange and blue-green were introduced to classify any overlap.

A number of approaches have been made to deal with the problem of overlapping colour space; from the implementation of multiple look up tables [6] to the implementation of 'maybe colours' [7]. Our method defers colour decision making when a colour value is shared between two objects. The overlapping shade is classified as the corresponding soft colour ('either' colour) until all colour information is processed. If a soft colour is suitably overlapping true colour information then the soft colour is used for additional object information (see Figure 1). The soft colour classification system has been implemented successfully for two years [5] of competition using a manual calibration system; however the classification principles can be applied to any calibration method.

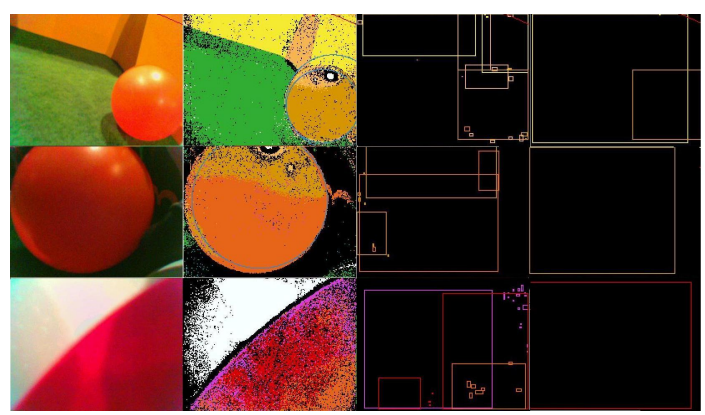

Fig. 1: Application of soft colour classification. Each row shows: Input images (taken at RoboCup 2006), colour classified image (using manually calibrated LUT), true and soft colour blobs formed and objects recognised.

\subsection{YUV and HSI Colour Space}

The YUV colour space is represented by; intensity (Y) and the colour information is given by chrominance values ( $\mathrm{U}$ and $\mathrm{V}$ ) which represent the blue-green correlation and red-green correlation. The HSI colour space is represented by; hue $(\mathrm{H})$ a value that represents the predominant wavelength of colour, saturation (S) a value for the amount of colour present and intensity (I) the darkness/brightness of the colour. By transforming the YUV image to HSI we can use hue as a convenient value for separating and labeling colour keys. Additionally the HSI colour space is intuitive, this allows for three dimensional solid regions of HSI colour space to be defined as a colour class.

\section{Method}

The method involves a number of stages, firstly images are selected, transformed to HSI and frequency data calculated (Figure 2). The S and I data can be divided 
into distinct regions for colours, black and white. The $\mathrm{H}$ data can be and the $\mathrm{H}$ frequency data is normalised for fitting Gaussian mixtures to separate colour information. Fitting of Gaussians to hue allows for upper and lower boundaries to be determined using standard deviations from the mean. Using soft colour principles the soft colour is applied to the hue values that overlap on the histogram. The upper and lower boundaries of H, S and I components are then used to fill in colour regions and convert from HSI regions to a YUV LUT to create solid classification.

\subsection{HSI Histograms}

We wanted a system that would be robust in the varied conditions that occur during a game; and also easily modifiable when a dramatic shift in colour space occurs (due to a change in location). Thorough manual calibration relies on image streams from hundreds to thousands of images long. From a stream of images we take a selection and convert from YUV to HSI. To reduce noise the images selected are of individual objects (colours) that fill the majority of the frame. Taking histograms of H,S and I channels (Figure 2) of test images allows for the colour space to be analysed. In the design process it was noticed that histograms of the hue channel showed distinct colour separation about the theoretical hue values of the object colours and a Gaussian mixture model could be applied to the frequency data. It also made apparent the problem colours and the sections of hue values that overlap.
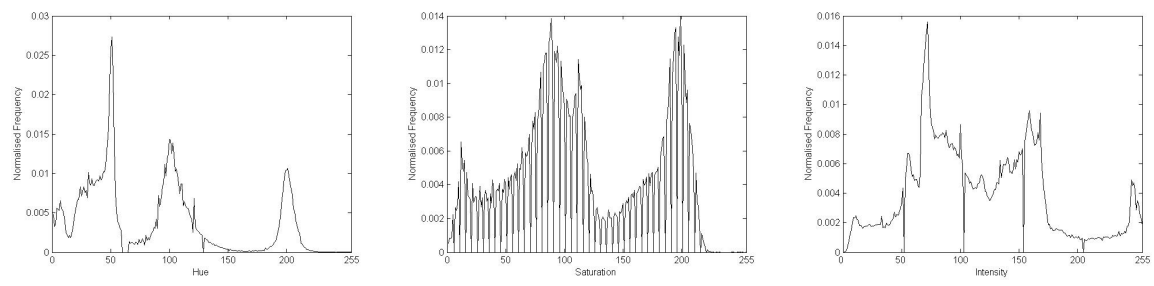

Fig. 2: Normalised histograms of hue, saturation and intensity channels from selected test images.

\subsection{Gaussian Mixtures}

We present research into segmenting in HSI using Gaussian mixtures in one dimension. Gaussian mixtures were chosen as opposed to other mixture models (e.g. t, Gamma, generalised $\lambda$ ) for its ease of extension to higher dimensions. The hue frequency data was chosen as the first dimension to apply the mixtures to as it holds important colour information and requires the most attention when segmenting. Future work involving the application of multidimensional Gaussian mixtures is outlined in Section 5.

Research involving Gaussian mixtures in robot vision is extensive and has included segmenting in YUV [8] and colour learning in HSI [9]. This paper 
presents the application of Gaussian mixtures to ensure hue values are unique to the object colour and to detect overlapping hue values. We are trying to find unique hue values for red, orange, yellow, green and blue. Any overlapping hue values is classed as soft colour. This is different to other systems in that it is not dependent on a hard boundary, only object colour uniqueness. This ensures the integrity of the system and allows for a practical implementation.

Using the normalised hue frequency data we used an optimisation approach to fitting a Gaussian mixture. The cost function of the optimisation was the sum of the squares error between the normalised (i.e. probability density) histogram and the Gaussian mixture density. These operations were performed in Matlab using the optimisation function fmincon. Since the object colour codes and their theoretical hue ranges are known we can define an initial values and constraints for the Gaussian parameters including the upper and lower boundaries of the hue means. The optimisation function outputs the parameter values; mean $\left(\bar{h}_{i}\right)$, standard deviation $\left(S_{i}\right)$ and probability $\left(p_{i}\right)$ for each colour present in the histogram. Using these we calculated hue ranges, $\bar{h}_{i} \pm k_{i} S_{i}$, where $k_{i}$ was initially interactively chosen on the basis of a histogram. Overlapping hue ranges were classified as soft colours. Figure 3 shows the optimised Gaussian mixture applied to the hue histogram data from Figure 2.

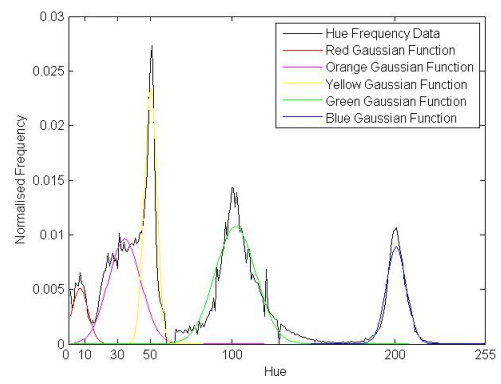

Fig. 3: Hue histogram and optimised Gaussian mixture to separate red, orange, yellow, green and blue.

\subsection{HSI Regions}

Creating solid regions in HSI colour space can be done by defining upper and lower boundaries for H,S and I channels. The optimisation function of the gaussian mixture outputs the mean and standard deviation for the hue values. This data is used to calculate bounday values. The number of standard deviations used for hue separation depends on the difficulty of separation of colours. For red, orange and yellow a value of one standard deviation from the mean is used to generate the upper and lower boundaries, for upper-yellow, green and blue hues are well separated standard deviations of two or three multiples are used. Saturation and intensity boundaries are interpreted from histograms as colour, black and white regions. The three dimensional region in HSI colour space can be 
labeled based on hue. Unique hue regions are labeled as the object colour whereas non unique hue values create the boundaries of soft colour regions. Black and white can be defined by saturation and intensity; both have low saturation but can be separated by their intensity values as they are located at either apex of the HSI cones. A larger region of intensity (brightness) values can be classified in this method due to the reliability gained from the separation of hue values. This is a step in illumination invariance [10] however a shift in colour temperature of lighting requires recalibration of hue Gaussians.

\section{Results}

The developed system had several notable results;

- Calibration time was greatly reduced.

- The number of required images was greatly reduced.

- The HSI regions resulted in solid classified images.

- Colours were successfully separated.

- Classification was robust and could handle change in lighting conditions.

The system has a significant reduction in calibration time from hours (approx $2 \mathrm{hrs}$ ) to minutes (approx $20 \mathrm{~min}$ ). Additionally the number of images required (for this example) was reduced from 1554 random images to 10 selective images.

The effect of HSI colour region classification compared to manual classification can be seen from Figure 4. By defining solid classified regions in HSI colour has resulted in 'solid' classification of images. It can also be seen that colours were sucessfully separated.

This is confirmed in Table 1. The table compares performance of classification of different objects. Blob formation rate was used as a measure of sucessful classification, object recognition rate is a measure of success of object recognition and false positive rate is a measure of blobs incorrectly recognised as objects. Comparing the data it can be seen that the success of classification is similar between both methods of classification. Also, it can be seen that an increase in object recognition rate leads to an increase in false positive rate. This is to be expected as often a compromise must be made between object detection and false positives.

It can be seen from Figure 4 that the system results in the successful separation of red and orange hues. This is of major importance in game situations. However every system has limitations, defining regions as rectangular may not be ideal and hence there is the need for extension to higher dimension Gaussian mixtures.

\section{Conclusion and Future Work}

Firstly this paper presented a practical colour classification method for fully separating colours, yet retaining complete colour information. Using this classification principle we have presented a solution to the hours of manual colour 


\begin{tabular}{|c|c|c|c|c|}
\hline Object & $\begin{array}{c}\text { Classification } \\
\text { Method }\end{array}$ & $\begin{array}{c}\text { Blob Formation } \\
\text { Rate }(\%)\end{array}$ & $\begin{array}{c}\text { Object Recognition } \\
\text { Rate(\%) }\end{array}$ & $\begin{array}{c}\text { False Positive } \\
\text { Rate(\%) }\end{array}$ \\
\hline Ball & Manual & 100 & 97.5 & 0.5 \\
\hline & HSI & 100 & 98.8 & 1.1 \\
\hline Yellow Goal & Manual & 99.6 & 97.1 & 0.0 \\
\hline & HSI & 98.0 & 97.4 & 0.0 \\
\hline Blue Goal & Manual & 100 & 98.0 & 0.0 \\
\hline & HSI & 100 & 98.4 & 0.0 \\
\hline
\end{tabular}

Table 1: Blob formation rate, object recognition rate and false positive rate for objects with different classification methods.

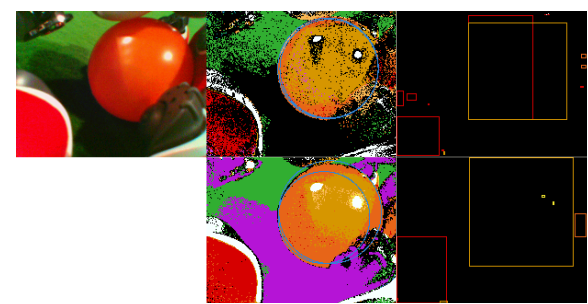

(a)

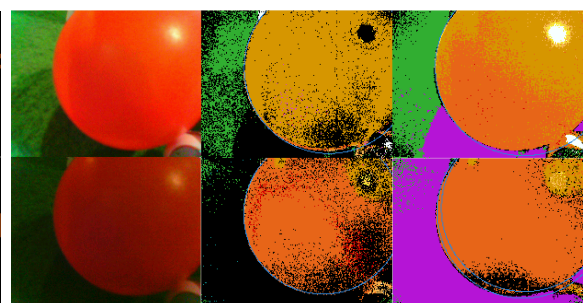

(b)

Fig. 4: (a)Separation of red and orange, manually calibrated LUT (first row) compared to HSI LUT (second row). (b) Robustness under varied lighting conditions, manually calibrated LUT (second column) compared to HSI LUT (third column).

calibration required during setup at RoboCup events. By transforming YUV images to HSI, computing histograms of each channel and applying gaussian mixtures to separate colour information we can define an upper and lower set of classification boundaries in HSI colour space. Then by transforming these HSI classified regions to a YUV look up table allows for an efficient calibration process that requires no additional processing to the original system. The developed solution resulted in increased reliability of object recognition due to the solid classified regions. However the reduction in misses has lead to an increase false positives. The system produced reliable results improved to that of a manually calibrated LUT whilst greatly reducing the number of required images and hence reduced image collection time, and greatly reducing the time involved with manually calibrating a LUT.

This method has proved to be successful but not optimal. Colour segmentation using Gaussian mixtures on hue alone is not sufficient in all cases. Our aim is to extend the use of Gaussian mixtures to higher dimensions. The extension of dimensions are to include saturation and intensity data and other statistically significant variables into the model to improve spatio-temporal consistancy (for example edge information, IR distance sensor information, etc ). To do this will 
require an alternate measure of goodness of fit such as maximum likelihood. Additionally we will investigate replacing the density based optimisation with either an Expectation Maximisation (EM) estimation for Gaussian mixtures or Bayesian mixture modelling.

\section{References}

1. Cameron, D., and Barnes, N.; Knowledge-Based Autonomous Dynamic Colour Calibration. D. Polani et al. (Eds.): RoboCup 2003: Robot Soccer World Cup VII, LNAI 3020, pp. 226-237 (2004).

2. Sridharan, M. and Stone, P.: Color Learning on a Mobile Robot: Towards Full Autonomy under Changing Illumination. In The International Joint Conference on Artificial Intelligence (IJCAI 07),Hyderabad, India. (2007)

3. Bruce, J., Balch, T. and Veloso, M.: Fast and inexpensive color image segmentation for interactive robots. In Proceedings of the 2000 IEEE/RSJ International Conference on Intelligent Robots and Systems (IROS-2000), vol. 3, pp. 2061-2066 (2000).

4. Austin, D.,Barnes, N.: Red is the new black - or is it? In: Proc. of the 2003. Australasian Conference on Robotics and Automation. (2003)

5. Qunilan, M. J. , Henderson, N., Nicklin, S. P., R. Fisher, , S. K. Chalup, R. H. Middleton, R. King "The 2006 NUbots Team Report.'University of Newcastle, EECS Technical Report, 2006.

6. Quinlan, M. J., Murch, C. L, Moore, T. G., Middleton, R. H., Yung Li, L. A., King, R. and Chalup, S. K,: The 2004 NUbots Team Report. University of Newcastle EECS Technical Report. (2004)

7. Stanton, C. and Williams, M-A.: A Novel and Practical Approach towards Color Constancy for Mobile Robots using Overlapping Color Space Signatures. To appear in the proceedings of the RoboCup 2005 Symposium. (2006)

8. Cohen, D., Hua Ooi, Y., Vernaza, P. and Lee, D. D.:The University of Pennsylvania Robocup 2003 Legged Soccer Team. Technical Report University of Pennsylvania. (2003)

9. D.A. van Soest, M. de Greef, J. Sturm and A. Visser, "Autonomous Color Learning in an Artificial Environment", Proc. 18th Dutch-Belgian Artificial Intelligence Conference, BNAIC'06, October 2006, Namen, Belgium (pp. 299-306).

10. Sridharan, M. and Stone, P.: Towards illumination invariance in the legged league. In RoboCup 2004: Robot Soccer World Cup VIII, ser. LNCS, D. Nardi, M. Riedmiller, and C. Sammut, Eds., vol. 3276. Berlin: Springer Verlag, pp. 196-208. (2005) 